$12-31-2016$

\title{
A New Distributed Chinese Wall Security Policy Model
}

\author{
Saad Fehis \\ Higher National School of Computer Algiers \\ Omar Nouali \\ Research Center on Scientific and Technical Information \\ Mohand-Tahar Kechadi \\ University College Dublin School of Computer Science and Informatics
}

Follow this and additional works at: https://commons.erau.edu/jdfs|

Part of the Computer Engineering Commons, Computer Law Commons, Electrical and Computer Engineering Commons, Forensic Science and Technology Commons, and the Information Security Commons

\section{Recommended Citation}

Fehis, Saad; Nouali, Omar; and Kechadi, Mohand-Tahar (2016) "A New Distributed Chinese Wall Security Policy Model," Journal of Digital Forensics, Security and Law. Vol. 11 : No. 4 , Article 11.

DOI: https://doi.org/10.15394/jdfsl.2016.1434

Available at: https://commons.erau.edu/jdfsl/vol11/iss4/11

This Article is brought to you for free and open access by

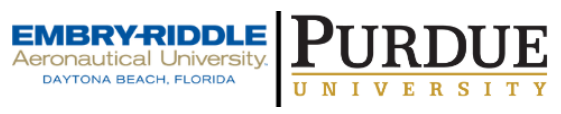
the Journals at Scholarly Commons. It has been accepted for inclusion in Journal of Digital Forensics, Security and Law by an authorized administrator of Scholarly Commons. For more information, please contact commons@erau.edu.

(c)ADFSL 


\title{
A NEW DISTRIBUTED CHINESE WALL SECURITY POLICY MODEL
}

\author{
Saad Fehis ${ }^{1}$, Omar Nouali ${ }^{2}$, and Mohand-Tahar Kechadi ${ }^{3}$ \\ ${ }^{1}$ Higher National School of Computer Algiers, Algeria s_fehis@esi.dz, \\ ${ }^{2}$ Research Center on Scientific and Technical Information CERIST, Algiers, \\ Algeria, onouali@cerist.dz, \\ ${ }^{3}$ University College Dublin School of Computer Science and Informatics, \\ Dublin, Ireland, tahar.kechadi@ucd.ie
}

\begin{abstract}
The application of the Chinese wall security policy model ( $C W S P M)$ to control the information flows between two or more competing and/or conflicting companies in cloud computing (Multi-tenancy) or in the social network, is a very interesting solution.

The main goal of the Chinese Wall Security Policy is to build a wall between the datasets of competing companies, and among the system subjects. This is done by the applying to the subjects mandatory rules, in order to control the information flow caused between them. This problem is one of the hottest topics in the area of cloud computing (as a distributed system) and has been attempted in the past; however the proposed solutions cannot deal with the composite information flows problem (e.g., a malicious Trojan horses problem), caused by the writing access rule imposed to the subject on the objects.

In this article, we propose a new CWSP model, based on the access query type of the subject to the objects using the concepts of the CWSP. We have two types of walls placement, the first type consists of walls that are built around the subject, and the second around the object. We cannot find inside each once wall two competing objects' data. We showed that this mechanism is a good alternative to deal with some previous models' limitations. The model is easy to implement in a distributed system (as Cloud-Computing). It is based on the technique of Object Oriented Programming (Can be used in Cloud computing "Software as a service SaaS") or by using the capabilities as an access control in real distributed system.
\end{abstract}

Keywords: Security Policy, Chinese Wall, Information flow, Distributed system, Cloud Computing

\section{INTRODUCTION}

The cloud computing technology comes with numerous advantages, but also brings with it some disadvantages or challenges. One of the challenges of cloud computing is the security, protection, and trust caused by Multitenancy. For instance, it is possible to find 
two competitors using the same cloud infrastructure and the same provider. This may cause issues of how to control the information flow between those competing companies and also between subjects in general. The Chinese Wall Security Policy $C W S P$ is a very interesting candidate solution to the above problem. The CWSP has already been used in commercial applications; for instance, the UK's financial sector, which provides consulting services, uses the CWSP model. As consultants must respect the confidentiality agreements, the CWSP is used to prevent such confidentiality from being breached by avoiding the information flow that causes conflict of interest between involved parties.

The CWSP was defined and named by Brewer and Nash (BN model). They have developed their first model in 1989 (Brewer \& Nash, 1989). The model became very attractive and therefore many other models based on the same idea have been proposed in subsequent years (Lin, 1989, 2000, 2002, 2003, 2007, 2015; Sharifi \& Tripunitara, 2013). The model and its variant have been successfully used in many applications (Atluri, Chun, \& Mazzoleni, 2004; Minsky, 2004; Hsiao \& Hwang, 2010; Wu, Ahn, Hu, \& Singhal, 2010; Tsai, Chen, Huang, Huang, \& Chou, 2011; Kesarwani et al., 2011; Xie, Ray, Adaikkalavan, \& Gamble, 2013) in order to control the information flow between the competing subjects residing in the same system. More importantly, after an extensive state-of-the-art on the proposed security models based on Chinese wall, we find that the main goal of those models is to control the composite information flow $(C I F)$ between competing companies (a malicious Trojan horse's problem), caused by the accesses in writing from the subjects on the objects. We can summarise these models by the following two important points:
- "The basis of the Chinese wall policy is that people are only allowed access to information which is not held to conflict with any other information that they already possess" (Brewer \& Nash, 1989). So, the user (subject) cannot access any other information in conflict with the information already possessed. So, each subject has a Granted and a Denied set of companies, where, each company in Granted set has their competing companies in the Denied set. The pairwise (Granted, Denied), interpreted by the build of the wall around the subject, named "subject's wall." And we conclude that, "we cannot find inside the same wall two competing objects' data."

- "Information can flow between two objects only via a subject, and information can flow between two subjects only via an object" (Sharifi \& Tripunitara, 2013), (malicious Trojan horse's problem). So, from these points in our hand, we can apply by symmetry the build of the wall around the object as a same rule to the subject (we cannot find in the same wall two competing objects' data) - So, we have the second wall around the object, named "object's wall."

However, the object is a passive entity, and is an opposite to the subject, considered it as an "active entity," has an "access right" Granted / Denied. The object is used to store the data, so each object has a "Stored right" (is not like the access right), where we cannot store inside the same object, data related to two competing companies. So, the object has two sets Allied and Conflict, where the Allied set has the companies (objects) who have a data stored inside the same object (so in allied), and the Conflict set contains the companies (objects) in conflict with the Allied set. The pairwise (Conflict, 
Allied) can be interpreted by the build of the wall around the object, named "object's wall."

In our approach, each subject has a security label composed by the pairwise (Granted, Denied), each object has a security label composed by the pairwise (Conflict, Allied) and the rule to execute the reading or writing query is "we cannot find two competing data inside the same wall (object's or subject's wall)." From this idea, we can build walls between the competing companies, and so the concept of the policy of Chinese wall (Brewer \& Nash, 1989). The model assures an efficient control of the direct and composite information flow.

At this level and to apply our model of the policy of CWSP in distributed system, the securities labels for the subject or object, can manage they, by the server that host the subject/object. So, each access query of any subject to any object will associate it with the security label of the subject, where it is verify by the server that host the object. So, our model can be viewed as a distributed CWSP model (D-CWSPM). And, also can be implemented based on a technique of Object Oriented Programming ( Can be used in Cloud computing "Software as a service SaaS") or by using the capabilities as an access control in real distributed system.

The remainder of the paper is organized as follows: In section 2, we will present our idea illustrated by an example. In section 3 , we will present the formal model and information flow problems. In section 4, we will present the D-CWSPM vs Access Matrix (can be used in system based on matrix as an access control). In section 5, we present the related previous works. In section 6 , we will present the implementation of D-CWSPM based on OOP. Then we will provide a conclusion with the future research directions, then in the appendix, the two main models (BN's and Lin's model) and the related of the binary relation, equivalence relation and the partitions. And finally, a list of references used in our work.

\section{MODELS IDEA ANALYSIS}

In this work, we based our idea on the access query type of the subject to the objects and the philosophy of the Chinese wall security policy CWSP. Its rule is the building of the walls between the competing companies. In our model, we have two types of walls placement, the first is built around the subject, and the second around the object. We cannot find inside the same wall two data related to two competing objects. So, we start by these analysis:

The subject firstly, is freely to choose to access to any object; at this step it's important to known the nature of this access: reading or writing access, as in following:

\subsection{Reading Queries:}

If the access is a reading request, or the subject reads from the object, so we can interpret this by the moving of the data from the object side into the subject side (inside the subject's wall). Therefore, there is a related data (information) of the object inside this wall. Consequently, the access is denied of this subject to the competing objects with the objects inside subject's wall. Therefore, the subject has two security labels:

- The Subject Wall Granted ( $S W G)$ : Is a set of objects, who theirs related data are in the subject's side.

- Subject Wall Denied $(S W D)$ : The set of all objects denied to moving theirs data into the subject's side.

The pairwise $(S W G, S W D)$, can be interpreted, by the building of the wall around the subject. 


\subsection{Writing Queries:}

If the access is writing query by the subject $S u b_{i}$ into the object $O b_{j}$, we can interpret this type of query by moving of objects related information from the $S u b_{i}$ 's wall into the object $O b_{j}$. So, the second wall is built around the object, and this object's wall cannot contains any competing object's data. Therefore, the object's wall has two security labels:

- The object's wall in Allied (OWA): A set of objects, where their related data stored inside $O b_{j}$. So, they are allied with the object $O b_{j}$.

- Object's wall in Conflict $(O W C)$ : A set of objects in conflict of interest with the objects, who have related data stored inside the object $O b_{j}$, so they denied to moving them into the $\mathrm{Ob}_{j}$ 's side.

The pairwise $(O W A, O W C)$, can be interpreted, by the building of the wall around the object $O b_{j}$.

\subsection{Illustration by an example:}

If we have two subjects $S u b_{1}$ and $S u b_{2}$, and five objects $O b_{1}, O b_{2}, O b_{3}, O b_{4}$ and $O b_{5}$, where $O b_{1}$ in competition with $O b_{2}, O b_{3}$ in competition with $O b_{4}$ but $O b_{5}$ neuter with the others objects (Table 1).

Table 1. The Initial State of the Object's Walls

\begin{tabular}{|c|l|}
\hline Object & Object's Wall \\
\hline$O b_{1}$ & $\mathrm{OWA}=\left\{O b_{1}\right\} ; \mathrm{OWC}=\left\{O b_{2}\right\}$ \\
$O b_{2}$ & $\mathrm{OWA}=\left\{O b_{2}\right\} ; \mathrm{OWC}=\left\{O b_{1}\right\}$ \\
$O b_{3}$ & $\mathrm{OWA}=\left\{O b_{3}\right\} ; \mathrm{OWC}=\left\{O b_{4}\right\}$ \\
$O b_{4}$ & $\mathrm{OWA}=\left\{O b_{4}\right\} ; \mathrm{OWC}=\left\{O b_{3}\right\}$ \\
$O b_{5}$ & $\mathrm{OWA}=\left\{O b_{5}\right\} ; \mathrm{OWC}=\{\emptyset\}$ \\
\hline
\end{tabular}

And we have the following sequence queries in our system:

Q1: Subject $S u b_{1}$ reading access from the object $O b_{1}$, so moving of data stored inside (or related to) of $O b_{1}$ to the subject side $S u b_{1}$. So we update the $S u b_{1}$ 's wall as following:

$$
S W G_{1}=\left\{O b_{1}\right\} \text { and } S W D_{1}=\left\{O b_{2}\right\},
$$

Because the object $O b_{1}$ in conflict with the object $\mathrm{Ob}_{2}$. And the subject's wall composed by the two pairwise $\left(S W G_{1}, S W D_{1}\right)$

Q2: Subject $S u b_{1}$ reading access from the object $\mathrm{Ob}_{2}$, this access is denied, because there is inside of its wall a data related to the object $O b_{1}$, where it is in competition with the object $\mathrm{Ob}_{2}$ (Rules of Chinese wall security policy (Brewer E Nash, 1989)).

Q3: Subject $S u b_{2}$ reading access from the object $\mathrm{Ob}_{2}$, so the moving of the data stored inside (or related to) the object $O b_{2}$. So we update the $S u b_{2}$ 's wall as following:

$$
S W G_{2}=\left\{O b_{2}\right\} \text { and } S W D_{2}=\left\{O b_{1}\right\},
$$

Because the object $\mathrm{Ob}_{2}$ in conflict with the object $O b_{1}$. And the subject wall composed by the two pairwise $\left(S W G_{2}, S W D_{2}\right)$

Q4: Subject $S u b_{1}$ reading access from the object $O b_{3}$, we have inside the $S u b_{1}$ 's wall a data related to the object $O b_{1}$ and this object isn't in conflict of interest with the object $\mathrm{Ob}_{3}$, so the access is granted and also the moving of the data from $O b_{3}$ to the subject $S u b_{1}$ side, inside of its wall. In the same time the access denied to the object $o b_{4}$, in conflict with $O b_{3}$. So we update of the $S u b_{1}$ 's wall as following: 


$$
\begin{gathered}
S W G_{1}=\left\{O b_{1}, O b_{3}\right\} \text { and } \\
S W D_{1}=\left\{O b_{2}, O b_{4}\right\} .
\end{gathered}
$$

Q5: Subject $S u b_{1}$ writing access to the object $O b_{5}$, so the moving data from the $S u b_{1}$ side into the object $O b_{5}$. It's clear, this data related to $\mathrm{Ob}_{1}$ or $\mathrm{Ob}_{3}$. So probably, the object $O b_{5}$ contains a data related to the objects $O b_{1}$ or $O b_{3}$, and cannot contains a two data related to two competing objects $\left(\mathrm{Ob}_{2}\right.$ in conflict with the object $\mathrm{Ob}_{1}$ and $\mathrm{Ob}_{3}$ with the object $\left.O b_{4}\right)$. So we need to update the $O b_{5}$ 's wall as follow:

$$
\begin{gathered}
O W A_{5}=\left\{O b_{5}, O b_{1}, O b_{3}\right\}, \\
O W C_{5}=\left\{O b_{2}, O b_{4}\right\} .
\end{gathered}
$$

The $O b_{5}$ 's wall composed by the pairwise $\left(O W A_{5}, S W C_{5}\right)$.

Q6: Subject $S u b_{2}$ writing access to the object $O b_{5}$, so moving data from its inside (a data related to $\mathrm{Ob}_{2}$ ) into the object $\mathrm{Ob}_{5}$. However, the object $\mathrm{Ob}_{5}$ contains a data related to the object $O b_{1}$ who it is in competition with $O b_{2}$ $\left(\mathrm{Ob}_{2} \in O W C_{5}\right)$. And, this is in contradiction with the Chinese wall security policy, so this query is denied and not permitted.

Let now, if we have a third subject $S u b_{3}$, where it need to read a data from the object $\mathrm{Ob}_{5}$ and then write it to the $\mathrm{Ob}_{2}$. So, the problem is that our malicious subject $\left(\mathrm{Sub}_{3}\right)$ need to create a CIF between competing object $O b_{1}$ and $O b_{2}$ !

Firstly, the subject $S u b_{3}$, read data from the object $\mathrm{Ob}_{5}$. The object $\mathrm{Ob}_{5}$, contains a data related to two objects $O b_{1}$ and $O b_{3}$ (from Q5) and also the object has two sets information the $O W A_{5}$ and the $O W C_{5}$. So, we have the two following steps:
1. The first step is the reading: After the reading access, we have inside $S u b_{3}$ 's wall a data related to three objects $O b_{1}$, $\mathrm{Ob}_{3}$ and $\mathrm{Ob}_{5}$. So, the updating of the subject's wall as following:

$$
\begin{aligned}
& S W G_{3}=S W G_{3} \cup O W A_{5}, \\
& S W D_{3}=S W D_{3} \cup O W C_{5}
\end{aligned}
$$

So, $O W C_{5}$ set contains $\mathrm{Ob}_{2}$.

2. The second step is the writing: the writing access to the object $\mathrm{Ob}_{2}$, this, is not permitted, because the object is in the Denied set $\left(S W D_{3}\right)$ of the subject. So, the access is denied.

So, as a result, our malicious subject cannot create a CIF between competing objects. And in end we can view in table 2 and 3, the final state of the Subjects' and Objects' walls.

Table 2. The End State of the Subject's Walls

\begin{tabular}{|c|l|}
\hline Subject & Subject's Walls \\
\hline$S u b_{1}$ & $S W G_{1}=\left\{O b_{1}, O b_{3}\right\}$ \\
& $S W D_{1}=\left\{O b_{2}, O b_{4}\right\}$ \\
$S u b_{2}$ & $S W G_{2}=\left\{O b_{2}\right\} ; S W D_{2}=\left\{O b_{1}\right\}$ \\
$S u b_{3}$ & $S W G_{3}=\left\{O b_{1}, O b_{3}, O b_{5}\right\} ;$ \\
& $S W D_{3}=\left\{O b_{2}, O b_{4}\right\}$ \\
\hline
\end{tabular}

\subsection{Queries' running conditions:}

Let the subject $S u b_{i}$ has two sets' object: the granted set $S W G_{i}$ and the denied set $S W D_{i}$, and the object $O b_{j}$ has two object's set: $O W A_{j}$ and $O W C_{j}$

After, the prior interpretation of the query access type (Reading / Writing), we can induce the mandatory condition to run the query of the access of the subject $S u b_{i}$ to the object $O b_{j}$ is: 
Table 3. The end state of the object's walls

\begin{tabular}{|c|l|}
\hline Object & Object's Walls \\
\hline$O b_{1}$ & $\mathrm{OWA}=\left\{O b_{1}\right\} ; \mathrm{OWC}=\left\{O b_{2}\right\}$ \\
$O b_{2}$ & $\mathrm{OWA}=\left\{O b_{2}\right\} ; \mathrm{OWC}=\left\{O b_{1}\right\}$ \\
$O b_{3}$ & $\mathrm{OWA}=\left\{O b_{3}\right\} ; \mathrm{OWC}=\left\{O b_{4}\right\}$ \\
$O b_{4}$ & $\mathrm{OWA}=\left\{O b_{4}\right\} ; \mathrm{OWC}=\left\{O b_{3}\right\}$ \\
$O b_{5}$ & $\mathrm{OWA}=\left\{O b_{1}, O b_{3}, O b_{5}\right\}$ \\
& $\mathrm{OWC}=\left\{O b_{2}, O b_{4}\right\}$ \\
\hline
\end{tabular}

"we cannot find inside the same wall, data related to two competing objects"

Formally as in the following:

$$
\begin{gathered}
S W G_{i} \cap O W C_{j}=\emptyset \text { and } \\
S W D_{i} \cap O W A_{j}=\emptyset
\end{gathered}
$$

\section{DISTRIBUTED CWSP MODEL:}

After, the prior illustration, we can now present the formal model.

Let:

- $O B=\left\{o b j_{1}, \ldots o b j_{n}\right\}$, denote the set of all objects,

- $S U=\left\{s_{1}, \ldots, s_{m}\right\}$, denote the set of all subjects,

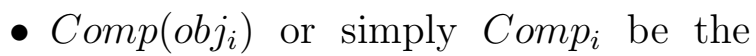
company dataset of object $o b j_{i}$.

\subsection{Dataset organization}

In our model we keep the dataset organization proposed by Lin (Lin, 1989)-(Lin, 2007), where:

- Lowest Level: we consider individual items of information, each concerning a single corporation. We will refer to the files in which such information is stored as objects (Brewer \& Nash, 1989).
- Intermediate level: we group all objects which concern the same corporation together into what we call a company dataset (Brewer \& Nash, 1989).

- Highest level: we associate with each company dataset, say X, a "Frechet neighborhood", denoted by CIN $(\mathrm{X})$ "Conflict of Interest Neighborhood of $\mathrm{X}$ ", where $\mathrm{CIN}(\mathrm{X})$ is the set of all company datasets that are in conflict of interest with $\mathrm{X}$.

\subsection{Conflict of interest relation CIR}

Let $C I R \subseteq O B \times O B$ as a binary relation, satisfies the following properties.

- CIR-1: CIR is symmetric.

- CIR-2: CIR is anti-reflexive.

It should be clear CIR-2 is necessary, a company cannot conflict to itself. If company $A$ is in conflicts with $B, B$ is certainly in conflicts with $A$, so CIR-1 is valid.

\subsection{Model}

Our model is 3 tuple (SU,OB, Query) where:

\subsection{1 $O B$}

Denote the set of all objects, where each object $o b j_{i}$ has or associated with two subsets of $O B$ :

- $O W A\left(o b j_{i}\right) \subseteq O B$, Or simply $O W A_{i}$, the set of all objects, where they have a related data stored inside the object $o b j_{i}$. If there is an object $o b j_{j} \in O W A_{i}$, so the object $o b j_{i}$ contains (or stored inside itself) a data related to the object $o b j_{j}$.

- $O W C\left(o b j_{i}\right) \subseteq O B$, Or simply $O W C_{i}$, the set of all objects denied to be stored 
their related data inside the object $o b j_{i}$. So, if there is an object $o b j_{j} \in O W C_{i}$, that, the object $o b j_{i}$ cannot will contain any related information of the object $o b j_{j}$. Otherwise, the object $o b j_{i}$ has a data related to another object in the conflict of interest with the object $o b j_{j}$.

And they are initially as following:

- $O W A\left(o b j_{i}\right)=o b j_{i}$, initialized by its self,

- $O W C\left(o b j_{i}\right) \quad=\quad\left\{o b j_{j} \in\right.$ $\left.O B \mid\left(o b j_{i}, o b j_{j}\right) \in C I R\right\}$

The pairwise $\left(O W A_{i}, O W C_{i}\right)$ can it interpret by the building of the wall around the object $o b j_{i}$. So, we cannot find inside the same wall, two data related to two distinct competing objects.

\subsection{2 $S U$}

Denote the set of all subjects, where each subject $S_{i}$ has or associated with two subsets of the object $O B$ :

- $S W G\left(S_{i}\right) \subseteq O B$, Or simply $S W G_{i}$, the set of the objects have a related data inside the subject wall of $S_{i}$ (read by the subject). So, if there is an object $o b j_{j} \in S W G_{i}$, so the subject $S_{i}$ contains a related data of the object $o b j_{j}$.

- $S W D\left(S_{i}\right) \subseteq O B$, Or simply $S W D_{i}$, the set of the objects denied to will be read by the subject $S_{i}$. So, if there is an object $o b j_{j} \in S W D_{i}$, that the subject $S_{i}$ cannot will contain (or read) any related data of the object $o b j_{j}$.

And they are initially as following:

- $S W G\left(S_{i}\right)=\emptyset$; initialized by an empty set, because the subject isn't yet read any object;
- $S W D\left(S_{i}\right)=\emptyset$ initialized by an empty set, because the subject is free to choose any object

The pairwise $\left(S W G_{i}, S W D_{i}\right)$ can it interpret by the building of the wall around the subject $S_{i}$, so, we cannot find inside the same wall two data related to two distinct competing objects.

\subsubsection{Query $\left(S_{i}, o b j_{j}\right.$, mode $)$}

Any query made by a subject $S_{i}$ to access to the object $o b j_{j}$ with the mode equal to:

- read: to read from the object

- write: to write into the object

The access is authorized, if and only if, this condition is verified:

$$
\begin{gathered}
S W G_{i} \cap O W C_{j}=\emptyset \text { And } \\
S W D_{i} \cap O W A_{j}=\emptyset
\end{gathered}
$$

And in the same time:

If the mode is Reading Query (Writing into the subject side, inside the subject's wall):

- $S W G_{i}=S W G_{i} \cup O W A_{j}$

- $S W D_{i}=S W D_{i} \cup O W C_{j}$

If the mode is Writing Query (In Object side, inside the wall that round the object):

- $O W A_{j}=S W G_{i} \cup O W A_{j}$

- $O W C_{j}=S W D_{i} \cup O W C_{j}$

Otherwise, the access is denied.

\section{INFORMATION FLOW'S AND D-CWSPM}

We give the following definition: 
DIF : A direct information flow between two companies $A$ and $B$ : denoted by $A \rightarrow B$, is a sequence of read query data from a company $A$ by any subject, then writing query of this data into the other company $B$ by the same subject.

CIF : A composite information flow from $A$ to $B$, is a sequence of DIFs (direct information flow) made by many subjects, which starts from $A$ and end at $B$ :

$$
A=A_{0} \rightarrow A_{1} \rightarrow \ldots \rightarrow A_{n}=B
$$

D-CWSPM's Theorem: The Distributed CWSPM assures that no DIF and no CIF between competing companies.

Proof: Let we have the two following propositions:

1. $(A, B) \in C I R \Rightarrow A \in O W C_{B}(B \in$ $\left.O W C_{A}\right)$ and $A \notin O W A_{B}\left(B \notin O W A_{A}\right)$ by symmetry.

2. There exists a CIF from $A$ to $B$, that is, a composite direct information flow of size $n$ :

$$
A=A_{0} \rightarrow A_{1} \rightarrow \ldots \rightarrow A_{n}=B
$$

We will use proof by Recurrence; on the number of DIFs between companies.

Let $n$ the number of DIFs between these two companies.

With $n=1$ :

First, the initial assertion: Since $A=$ $A_{0} \rightarrow A_{1}=B$ is a DIF, or read data from object $A_{0}$ by any Subject $S_{i}$, then write it by the same subject to $A_{1}=B$ :

- Reading by the subject $S_{i}$ from the object $A_{0}$ is granted, if and only if:

$$
\begin{gathered}
S W G_{i} \cap O W C_{A_{0}}=\emptyset \text { And } \\
S W D_{i} \cap O W A_{A_{0}}=\emptyset
\end{gathered}
$$

And the result is:

$$
\begin{gathered}
S W G_{i}=S W G_{i} \cup O W A_{A_{0}} \\
\text { and } \\
S W D_{i}=S W D_{i} \cup O W C_{A_{0}}
\end{gathered}
$$

- Writing by the subject to object $A_{1}$ is granted, if and only if:

$$
\begin{gathered}
S W G_{i} \cap O W C_{A_{1}}=\emptyset \text { And } \\
S W D_{i} \cap O W A_{A_{1}}=\emptyset .
\end{gathered}
$$

This condition, assures that $A_{0}$ isn't in the conflict of interest with $A_{1}$. However, in our case we have, $A=A_{0}$ in conflict with $A_{1}=B$ ( $A$ in conflict with $B$ ), so no DIF between $A$ and $B$ if they are in competing, so the query is denied QED.

So, if $n$ equal to 1 , there isn't a DIF between two competing companies.

Otherwise, in the case of $A_{0}$ isn't in conflict of interest with $A_{1}$ :

$$
\begin{aligned}
& O W A_{1}=O W A_{1} \cup S W G_{i} \\
& O W C_{1}=O W C_{1} \cup S W D_{i} .
\end{aligned}
$$

So the result of the DIF from $A_{0}$ to $A_{1}$ is:

$$
\begin{aligned}
& O W A_{0} \subseteq O W A_{1} \\
& O W C_{0} \subseteq O W C_{1} . \\
& \text { So, } A_{0} \in O W A_{1} .
\end{aligned}
$$

Let now our theorem is true with $n-1$ DIFs and we need to verify, if is it true for $n$ DIFs. So we have:

$$
A=A_{0} \rightarrow A_{1} \rightarrow \ldots \rightarrow A_{n-1}
$$

And we need to extend it to $A_{n}=B$, where $A \in O W C_{B}$. From the sequence of size $n-1$ of the DIFs, we have:

$$
\begin{gathered}
A=A_{0} \in O W A_{0} \subseteq O W A_{1} \subseteq O W A_{2} \subseteq \\
\ldots \subseteq O W A_{n-1} .
\end{gathered}
$$


And it is the same with the set of $O W C$.

Let, there is a subject $S_{j}$ need to create a DIF between $A_{n-1}$ and $A_{n}$. So, the subject $S_{j}$ needs to read from $A_{n-1}$ then writing to $A_{n}$, so we have two following steps:

- The first step is reading from $A_{n-1}$ by the subject and the consequence is:

$$
\begin{gathered}
S W G_{j}=S W G_{j} \cup O W A_{n-1} \\
\text { And } \\
S W D_{j}=S W D_{j} \cup S W C_{n-1} .
\end{gathered}
$$

So $A \in S W G_{j}$ and $B \in O W C_{A} \subseteq$ $S W D_{j}$.

- The second step is writing to $A_{n}$, with the condition, that $S W G_{j} \cap O W C_{n}=\emptyset$. However, in our case, we have $A \in$ $S W G_{j}$ and $A \in O W C_{n}$, so the intersection is different from the empty set $(\emptyset)$.

So, the writing query is denied because $A_{0} \in O W C_{n}$. And the result is that no CIF between those competing companies $A$ and $B$. QED

We conclude, that our distributed DCWSPM assures that no CIF between any two companies if they are in conflict of interest.

\section{D-CWSPM VS ACCESS MATRIX}

In this section, we will present how to implement our distributed model using the matrix as mechanism, and to compare it with the previous proposed models.

\subsection{Access matrix Model}

Firstly, in our model, any object's wall is represented by the pairwise $(O W A, O W C)$. And The set of walls can be viewed as a binary relation between objects, therefore can be represented by a matrix, where we call it, the object's wall matrix $(O W M)$.

The $O W M$, be a matrix with element $O W M(i, j)$ corresponding to the members of $O B \times O B$, where the value of $O W M(i, j)$ is:

1: The object $o b j_{i}$ contains (or stored inside itself) a data related to $o b j_{j}$;

0: The object $o b j_{i}$ cannot contain any data related to the object $o b j_{j}$. Or the object $o b j_{i}$ has a data related to an object in the conflict of interest with the object $o b j_{j}$.

-1: There isn't any data related to the object $o b j_{j}$ or to its competing objects, stored inside the object $o b j_{i}$;

Initially,

- $O W M(i, j)=1$ if $i=j$,

- $O W M(i, j)=0$ if $\left(o b j_{i}, o b j_{j}\right) \in C I R$,

- otherwise -1 .

And we can define also two subsets of $O B$ :

- $O W A\left(o b j_{i}\right) \quad=\quad\left\{o b j_{j} \in\right.$ $O B \mid O W M(i, j)=1\}$ the set of all objects, have their data stored inside of the object $o b j_{i}$,

- $O W C\left(o b j_{i}\right) \quad=\quad\left\{o b j_{j} \in\right.$ $O B \mid O W M(i, j)=0\}$ the set of all objects denied to be stored inside of the object $O_{i}$,

The second wall in our model is the subject's wall. So for any subject $S_{i}$, its wall can be represented by, the pairwise $\left(S W G_{i}, S W D_{i}\right)$. So the set of the walls can be represented by a binary relation between subject and object, and can be represented by a matrix, where we call it, the Subject's wall matrix $(S W M)$. 
The $S W M$ : is an access matrix with elements $S W M(i, j)$ corresponding to the members of the $S U \times O B$, where the value of $S W M(i, j)$ is:

1: The subject $S_{i}$ contains a data related to the object $o b j_{j}$.

0: The subject $S_{i}$ cannot contain any related information of the object $o b j_{j}$

-1: There isn't any data related to the object $o b j_{j}$ or their competing objects, inside of the subject $S_{i}$;

Initially, $S W M(i, j)=-1$ for all $(i, j)$.

And From this matrix, we can also define two subsets of:

- $S W G\left(S_{i}\right)=\left\{O_{j} \in O B \mid S W M(i, j)=\right.$ $1\}$ the set of the objects, have a data inside of the subject wall of $S_{i}$,

- $S W D\left(S_{i}\right)=\left\{O_{j} \in O B \mid S W M(i, j)=\right.$ $0\}$ the set of the objects denied to the subject $S_{i}$,

In the end, and after the representation of the objects' wall and the subjects' wall by using the matrix ( $O W M, S W M)$ as mechanism, we will show, how updating them, for every access query.

Let the Query $\left(S_{i}, o b j_{j}\right.$, mode $)$, any query made by a subject $S_{i}$ to access to the object $o b j_{j}$ with the mode equal to read or write. The access is authorized, if and only if; this condition is verified:

$$
\begin{gathered}
S W G_{i} \cap O W C_{j}=\emptyset \text { And } \\
S W D_{i} \cap O W A_{j}=\emptyset
\end{gathered}
$$

And in the same time:

If the mode is Reading Query (In Subject side): So the updating of the subject's wall as following:

- $S W M(i, h)=1$ where $o b j_{h} \in O W A_{j}$, (as $S W G_{i}=S W G_{i} \cup O W A_{j}$ )
- $S W M(i, h)=0$ where $o b j_{h} \in O W C_{j}$, (as $S W D_{i}=S W D_{i} \cup O W C_{j}$ )

If the mode is Writing Query (In Object side): So the updating of the object's wall as following:

- $O W M(j, h)=1$ where $o b j_{h} \in S W G_{i}$, ( as $O W A_{j}=S W G_{i} \cup O W A_{j}$ )

- $O W M(j, h)=0$ where $o b j_{h} \in S W D_{i}$, (as $O W C_{j}=S W D_{i} \cup O W C_{j}$ )

Otherwise, the access is denied, and in the same times:

- $S W M[i, j]=0$

\subsection{Information flows, Objects, Companies and their Allies}

In our model, we focused our work, on the relations between objects and the information flows between them. However, what about of the flow between the objects in the same company?

The answer is the information flows is freely between them. So, we build the object wall around the allied objects (allied companies).

However, in our proposed model the update of the matrix $O W M$ is focused on the object, and not on the companies. So, to fix this problem, the solution is the mapping of $O W M$ from of $O B \times O B$ to Comp $\times C o m p$. By this mapping, we will assure that, we can't find the data of two competing companies stored inside the same company (in different objects of the same company).

\section{RELATED WORKS}

In this section we will present a set of related works, where in the first section we present the previous proposed models based an access matrix, and theirs problems. Then, 
in the next section we will present the related application works of the CWSP in the environment as distributed system (CloudComputing, work-flow).

\subsection{Related works based on access matrix}

The CWSPM was identified and so named by Brewer and Nash (BN's model), where they developed a mathematical model for this policy (Brewer \& Nash, 1989). Their idea is the grouped of the dataset of companies in conflict of interest classes $(C O I)$, so a set of partitions and applying to subjects a mandatory ruling, where all subjects are allowed access to at most one dataset belonging to each such conflict of interest class (security rule). Where, the access is only granted if the object requested:

a) Is in the same Company Dataset as an object already accessed by that subject, or,

b) Belongs to an entirely different Conflict of Interest Class.

Access, means read or write. So, this to answer that no direct information flow $(D I F)$ between competing companies. And also they prevents the CIF by the application of the start-property rule to write access, where is only permitted if, the:

a) Access is permitted by the simple security rule, and,

b) No object can be read which is in a different Company Dataset to the one for which write access is requested and contains un-sanitized information.

The proposal was a great idea. Unfortunately, BN's model was based on incorrect assumption that corporate data can be partitioned (decomposed) into mutually disjoint conflict of interest classes (COIclasses), such a disjoint collection is called a partition in mathematics. COI-classes seldom disjoint, they do overlap, and hence BN theory collapses. Also, the authors did not distinguish between human users and subjects that are processes running on behalf of users. The BN's write rule (*-property) is successful in preventing such information leakage by Trojan Horse. However, it does so at an unacceptable cost.

It is easy to see that the $\mathrm{BN}$ write rule has the following implications (Sandhu, 1992): A subject which has read objects from two or more company datasets cannot write at all. And, a subject which has read objects from exactly one company dataset can write to that dataset. These implications are clearly unacceptable (if the computer system is to be used for something more than a read-only repository of confidential information)(Sandhu, 1992). Under this regime a consultant can work effectively so long as he or she is assigned to exactly one company (however, even then the consultants is forbidden to write public information). When the consultant is assigned to a second company, he or she will be unable to write any information into the system. Consequently, the model proposed is very restrictive as it allows a consultant to work for one company.

Sandhu (Sandhu, 1992) improves upon this model by making a clear distinction between users, principals, and subjects, defines a lattice-based security structure, and shows how the CWP complies with the BellLapadula model (Bell \& La Padula, 1976).

In the same year, Lin announces a modified model, called an aggressive Chinese Wall Security Policy Model $(A C W S P M)($ Lin, 1989) to fix the errors of BN. The error is that the conflict of interest $(C O I)$ is a binary relation $(C I R)$, and not, an equivalence class (partitions). The CIR is non-reflexive, symmetric and Anti-transitive. The Lin's idea is 
the construction of a partition, so an equivalence relation. Lin, extend the CIR relation to an equivalence relation (partition). However, then the known properties of a binary relation do not support the elegance and crispness of an equivalence relation, the enthusiasm was lost.

Based on the work of Pawlak (Pawlak, 1984) (Pawlak, 1997), Lin in (Lin, 2002), show that the lack of crispness of the ACWSP, since CIR cannot produce a partition. However, the partition, can be recaptured by the induced equivalence relation of a binary relation. The idea is "each binary relation induced equivalence relation" (Lin, 2002)-(Lin, 2007). The induced equivalence relation named by IAR "In allied with" relation used by Lin is the complement of CIR (Theorem: CIR is a symmetric and antireflexive and anti-transitive binary relation. Its complement IAR is an equivalence relation).

In the end Lin applied a rules to subjects based on CIR relation and an allied partition, all this to answer that no information flow can will occur between the competing companies.

From these previous models, we are observing, that both models BN and Lin, based on the partitioning of the companies in set of class (partitions). In BN's model the competing companies in the same partition, however, in Lin's model the allied companies in the same partition. The partitions in BN's model can overlap (example $A$ in conflict with $B, B$ with $C$ and $C$ with $D$ ). In Lin's model the partition based on the complement of the CIR relation (induced equivalence relation), can overlap if the CIR relation is not "ant-transitive", case named by Lin as a "Bad CIR Relation" (page 10 in (Lin, 2003)), so a real case excluded by Lin's model!

Also, we are observing, that, Lin in (Lin, 1989)-(Lin, 2007) fix the problems of the DIF / CIF by the using of the same BN's idea (Brewer \& Nash, 1989), unsanitized/sanitized information and also the $\mathrm{read} /$ write access type, where: In $\mathrm{BN}$ the unsanitized information is confined to its selfcompany but in Lin to allied dataset. So, the inheriting of the problem (Sandhu, 1992) "consultant to work for one company only" to "consultant to work for one allied companies only". So, no difference between the problem of the DIF and the CIF inside the same company / allied companies (as a set of objects in the same of a single company).

There is a recent and interested work proposed by Sharifi and al. (Sharifi \& Tripunitara, 2013), where they proposed a LeastRestrictive Enforcement of the CWSPM based on graph representation. Their enforcement mechanism mediates read attempts only to prevent subject-violations, and write attempts only to prevent object-violations. However, there is a strong mathematical confusion between the notion of the class, partition, equivalence binary relation and the transitivity property (page 3). Also, their graph representation is very complex for the implementation.

Finally, our new model in this article is easy for the implementation based access matrix and fixing the problems of the previous proposed models.

In the first, our main objective is the application of the CWSP in the Cloud Computing, and the social network and not the proposition of a new model for the CWSP. However, and after analyses of the previous proposed models (Brewer \& Nash, 1989)-(Sandhu, 1992), and theirs applications (Atluri et al., 2004) (Hsiao \& Hwang, 2010) (Wu et al., 2010) (Tsai et al., 2011) (Kesarwani et al., 2011) (Xie et al., 2013) (Alqahtani, Gamble, \& Ray, 2013) (Minsky, 2004), we are surprising by many problems. For example the problem of the Conflict of interest (COI) is a set of disjoint class or a 
binary relation (CIR), the error was fixed in 1989 by Lin (Lin, 1989)-(Lin, 2007), but to our days there are many applications based on COI classes and not a CIR binary relation.

\subsection{Related application works in environment as distributed system:}

In the past, there are many attempt to applying the CWSPM in environment as distributed system.

Firstly in the Cloud-Computing (as a distributed system), The CWSP used in (Tsai et al., 2011), is to fix inter-VM attack from competitors, which targets at the VMs running on the same physical machine, so each two competing VMs cannot hosted in the same physical machines so that physical isolation. The authors use the conflict of interest and the graph colouring algorithm for the VM deployment. However, the authors were based on centralized control mechanism.

Also, in (Wu et al., 2010) use the CWSPM for the "Information Flow Control in Cloud Computing", at the IaaS level.Based on the concept of the conflict of interest is partition (BN's model), however, it is a binary relation (Lin, 1989), and so they based on wrong model, and the same problem with two other work (Kesarwani et al., 2011)-(Xie et al., 2013) in Cloud Computing.

In the end, there is an interesting work (Minsky, 2004), named by "A Decentralized Treatment of a Highly Distributed ChineseWall", however, they based on wrong model, the Brewer and Nash model (Brewer \& Nash, 1989).

\section{IMPLEMENTATION D-CWSPM BASED ON OBJECT ORIENTED PROGRAMMING}

To valid our approach, we developed a prototype, based on Object Oriented Programming (OOP), and using the $\mathrm{C}++$ as programming language. In this model's implementation, we defined, four following classes:

- System Class: This class is the main class of the implementation, where each instance from this class, contains in its private section, the companies' identification, the CIR Relation definition and the subjects' identification in the system. The instance, is responsible for the creation/destruction of the companies and the updating of the CIR Relation.

- Company Class: This class is the set of all companies, where each company's instance contains the following proprieties: The identification list of all its objects and the company's wall, the pairwise $(C W A, C W C)$. The company instance responsible for the creation / destruction of their objects.

- Object Class: This class is the set of all objects in our system, where each instance is related to a single company, and has its "object wall", the pairwise $(O W A, O W C)$. The class has also a set of interface for the communication with the other object in the system.

- Subject Class: This class is the set of all subject in our system, where each instance has an single identification, and can access to any object in our system, by using the object interfaces, and CWSP rule. 


\section{CONCLUSION}

The Chinese wall security policy model $(C W S P M)$ is very interesting solution, to control the information flow between competing companies in cloud computing (multitenancy) or social network (as a real competing platform) in general. The CWSPM's idea is the building of the wall between the dataset of the competing companies by applying of a mandatory rules to the subjects (people are only allowed access to information which is not held to conflict with any other information that they already possess (Brewer \&5 Nash, 1989)).

So, the CWSPM can be used as mechanism to control the information flow, and in the same time as an access control imposed to the subjects. However, the previous proposed model have a many problems (Brewer \& Nash, 1989)-(Lin, 2007), (Sharifi \& Tripunitara, 2013), with application of these model in different way, with the same problems, and based on a matrix as mechanism without distribution.

In this work, we proposed a new model for this policy, where we named it a Distributed Chinese Wall Security policy Model $(D-C W S P M)$. Our model is real interpretation of the CWSP, (we can't find inside the same wall a data related to competing companies). The D-CWSPM's idea is the building of the wall around the subject (Brewer \& Nash, 1989), as the same to the objects. The model fix the problem of the "a malicious Trojan horses", based on the concept of "the information can flow between two objects only via a subject and information can flow between two subjects only via an object (Sharifi \& Tripunitara, 2013)".

Our model is based on a mathematical model, where the Conflict of interest is a binary relation and not a set of partitions (Class) (Lin, 1989)-(Lin, 2007). And, by the interpretation gave of two kinds of the queries (Reading / Writing). Our model assures that we cannot find any information flow between two competing companies, Direct or Composite information flow. So the fixing of the malicious Trojan horses problem.

Our model is easy to implementing it, in any way, basing on an access matrix between subjects/objects (can be compared with the previous proposed models) or in real distributed system (as Cloud Computing).

We have validate our model, by an implementation prototype, based on the technique of, Object Oriented Programming. Where, the entire security labels are distributed among the system's elements ( $o b$ ject and subject). Which, any element had its security label (its Wall). This, prototype, can will be applied it, in the CloudComputing (Software as a service SaaS) or in the social network as real competing environment.

In the future works,

1. Initially, our main objective was the application of the CWSPM in the CloudComputing and not the proposition of a new model. So, our next step is the application of this model in the CloudComputing at the service level "Infrastructure As a Service (IaaS)", to control the information between Virtual machines (multi-tenancy). Then the application of the model at the service level "Software As a service (SaaS)", by the developing of prototypes, based on the technique of Object Oriented Programming.

2. Introduction of this security model in the conception and development process of the solutions' kind as SaaS. So, the PaaS level (Platform as a Service).

3. We believe to applying our model in any previous application of the Chinese wall 
in the past based on the wrong models.

4. The model can be used in Inter-process communication $(I P C)$, so not always and only between subject and object. So to extending it between processes (subjects) as active entity in the same system.

5. In the end, the implementation of our model by using the capabilities as an access control in real distributed system, and to control the information flow between competition groups in the social network.

\section{APPENDICES, PREVIOUS MODELS}

In this section we will present the two main proposed model of CWSP, the BN's and Lin's model

\subsection{BN's Model:}

\subsubsection{Database organisation}

In the BN's model, all corporate information is stored in a hierarchically arranged filing system such as that shown in Figure1.

- At the lowest level, we consider individual items of information, each concerning 3 single corporation. In keeping with BLP, we will refer to the files in which such information is stored as objects; There are three levels of significance:

- At the intermediate level, we group all objects which concern the same corporation together into what we will call a company data set;

- At the highest level, we group together all company datasets whose corporations are in competition. We will refer to each such group as a conflict of interest class.

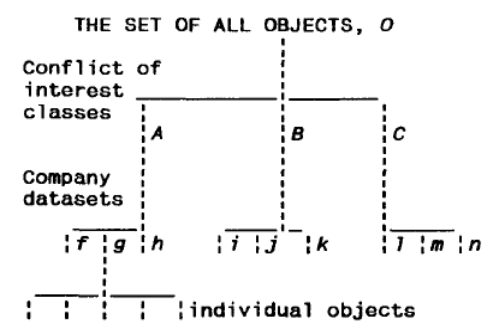

Figure 1. The composition of the objects (Brewer \& Nash, 1989)

\subsubsection{Basic Model}

Let $S$ be a set of subjects, $O$ be a set of objects and $L$ a set of security labels $(x, y)$. One such label is associated with each object. We introduce functions $X(o)$ and $Y(o)$ which determine respectively the $x$ and $y$ components of this security label for a given object $O$. We will refer to the $x$ as conflict of interest classes, the $y$ as company datasets and introduce the notation $x_{j}, y_{j}$ to mean $X\left(O_{j}\right)$ and $Y\left(O_{j}\right)$ respectively. Thus for some object $O_{j}, X_{j}$ is its conflict of interest class and $y_{j}$ is its company dataset.

- Axiom l:

$$
y_{1}=y_{2} \rightarrow x_{1}=x_{2}
$$

In other words, if any two objects $0_{1}$ and $\mathrm{O}_{2}$ belong to the same company dataset then they also belong to the same conflict of interest class.

\section{- Corollary 1:}

$$
x_{1}<>x_{2} \rightarrow y_{1}<>y_{2}
$$

In other words, if any two objects $0_{1}$ and $\mathrm{O}_{2}$ belong to different conflict of interest classes then they must belong to different company datasets. 


\section{- Definition l:}

$N$, a boolean matrix with elements $N(v, c)$ corresponding to the members of $S \times C$ which take the value true if subject $s_{v}$ has, or has had, access to object $O_{c}$ or the value false if $S_{v}$ has not had access to object $O_{0}$ Once some request $R(u, r)$ by subject $S_{u}$ to access some new object $O_{r}$ has been granted then $N(u, r)$ must be set true to reflect the fact that access has now been granted. Thus, without loss of generality, any request $R(u, r)$ causes a state transition whereby $N$ is replaced by some new $N$, $N^{\prime}$.

\section{- Axiom 2:}

Access to any object $O_{r}$ by any subject $s_{u}$ is granted if and only if for all $N(u, c)=$ true (i.e. by D1, $s_{u}$ has had access to $O_{o}$ )

$$
\left(\left(X_{o}<>X_{r}\right) \text { or }\left(y_{c}=y_{r}\right)\right) .
$$

\section{- Axiom 3:}

$N(v, c)=$ false, for all $(v, c)$ represents an initially secure state.

\section{- Axiom 4:}

If $N(u, c)$ is everywhere false for some $s_{u}$ then any request $R(u, r)$ is granted.

\section{- Theorem 1:}

Once a subject has accessed an object the only other objects accessible by that subject lie within the same company dataset or within a different conflict of interest class.

\section{- Theorem 2:}

A subject can at most have access to one company dataset in each conflict of interest class.

\section{- Theorem 3:}

If for some conflict of interest class $X$ there are $X_{v}$ company datasets then the minimum number of subjects which will allow every object to be accessed by at least one subject is $X_{v}$.

\subsubsection{Sanitized Information}

\section{- Definition 2:}

For any object $O_{a}$,

$Y_{a}=Y_{o}$ implies that $O_{a}$ contains sanitized information

$Y_{a}<>Y_{o}$ implies that $O_{a}$ contains unsanitized information

\section{- Axiom 5:}

$$
Y_{o} \Longleftrightarrow X_{o}
$$

In other words, if an object bears the security label $Y_{o}$ then it must also bear the label $X_{o}$ and vice versa. theorem 2 tells us that all subjects can access this company dataset.

\section{- Axiom 6:}

Write access to any object $O_{b}$ by any subject $S_{u}$ is permitted if and only if $N^{\prime}(u, b)=$ true and there does not exist any object $O_{a}\left(N^{\prime}(u, a)=\right.$ true $)$ which can be read by $S_{u}$ for which:

$$
Y_{a}<>Y_{b} \text { and } Y_{a}<>Y_{o} .
$$

\section{- Theorem 4:}

The flow of un-sanitized information is confined to its o,wn company dataset; sanitized information may however flow freely throughout the system.

\subsection{Lin's Model:}

In this section and before to present the Lin's model we start by some by presenting some properties related to the binary relations, then the Lin's model. 


\subsubsection{Binary Relation Property}

Let $V$ a set of objects (or elements), and we recall some definitions:

- A binary relation is a subset, $B \subseteq V \times V$ for each object $p \in V$, we associate a set $B_{p}$ defined by:

$B_{p}=\{v \in V \mid p B v\}$ or $B_{p}=\{v \in$ $V \mid(p, v) \in B\}$.

That consists of all elements $v$ that are related to $p$ by $B$. $B_{p}$ is called a binary neighbourhood.

If the binary relation is an equivalence relation, then $B_{p}$ is the equivalence class containing $p$.

- A symmetric binary relation $B$ is a binary relation such that for every $(u, v) \in$ $B$ implies $(v, u) \in B$.

- A binary relation $B$ is anti-reflexive: if $B$ is non-empty and no pair $(v, v)$ is in $B$. That is, $B \cap \Delta=\emptyset$, where $\Delta=$ $\{(v, v) \mid v \in V\}$ is called diagonal set.

- A binary relation $B$ is anti-transitive: if $B$ is non-empty and if $(u, v)$ belongs to $B$ implies that for all $w$ either $(u, w)$ or $(w, v)$ belongs to $B$.

Let the complement, $B^{\prime}=V \times V \sim$ $B$, is called the complement binary relation (CBR) of $B$.

Proposition: if $B$ is symmetric, antireflexive and anti-transitive, then $B^{\prime}$ is an equivalence relation (Lin, 2007).

Corollary 4: If $B$ is symmetric, antireflexive and anti-transitive, then $B^{\prime}$ is the induced equivalence relation $E_{B}$.

\subsubsection{Model}

In spite of their error, Brewer and Nash's intuitive idea is a fascinating one. To keep their spirit, in (Lin, 1989) Lin reformulated the model based on a general binary relation; however, the expected sharpness and crispness of the model, which are reflections some characteristics of equivalence relations, are lost. With the notion of the induced equivalence relation, in this section, we will present the mains points of Lin's model based on induced equivalence.

Let $O$ be a set of objects; an object is a dataset of a company. In Lin's model the conflict of interest is a binary relation, noted by $C I R$. Where $C I R \subseteq O \times O$, satisfies the following properties:

CIR-1: CIR is symmetric.

CIR-2: CIR is anti-reflexive.

CIR-3: CIR is anti-transitive.

It should be clear CIR-2 is necessary; a company cannot conflict to itself. If company $A$ is in conflicts with $B, B$ is certainly in conflicts with $A$, so CIR-1 is valid.

To see CIR-3, let $O=\{U S A, U K, U S S R\}$ be a set of three countries. Let $C I R$ be "in cold war with". If the relation "in cold war with" were transitive, then the following two statements:

(1) USA is in cold war with USSR.

(2) USSR is in cold war with UK.

would imply that

(3) USA is in cold war with UK.

Obviously, this is absurd. In fact this argument is applicable to any country; In other words, (2) and (3) cannot be both true for any country (that replaces UK). So we have anti-transitivity for CIR.

Let $E_{C I R}$ be the induced equivalence relation of CIR. In this model a new "axiom" will be explicitly added, though it is implied by the others (See Proposition 2)

CIR-4: The granulation of CIR and partition of $E_{C I R}$ are compatible, in the sense that each CIR-neighbourhood is a union of $E_{C I R^{-}}$equivalence classes. 
In (Lin, 1989), So, the placed the Chinese walls on the boundary of a CIRneighbourhood, this "new axiom" implies that that such a boundary is actually on some boundary of some unions of ECIRequivalence classes.

CIR-5: If we interpret CIR as "in cold war with" - relation, then the complement is "in ally with"-relation (IAR). IAR is an equivalence relation, by Corollary 4 .

Here are the same views of theorems in (Brewer \& Nash, 1989) and (Lin, 1989).

\section{- Theorem 1:}

Once a agent $S_{i}$ has accessed an object $O_{j}$, the only other objects $O_{k}$ accessible by $S_{i}$ is either inside the allied dataset of $O_{j}$ or outside of $C I R_{O_{j}}$.

\section{- Theorem 2:}

The minimum number of agents which allow every object to be accessed by at least one agent is $n$, where $n$ is the number of $E_{C I R^{-}}$equivalence classes.

\section{- Theorem 3:}

The flow of un-sanitized information is confined to its allied dataset; sanitized information may, however, flow freely through the system. 


\section{REFERENCES}

Alqahtani, S. M., Gamble, R., \& Ray, I. (2013). Auditing requirements for implementing the chinese wall model in the service cloud. In Services (services), 2013 ieee ninth world congress on (pp. 298-305).

Atluri, V., Chun, S. A., \& Mazzoleni, P. (2004). Chinese wall security for decentralized workflow management systems. Journal of Computer Security, 12(6), 799-840.

Bell, D. E., \& La Padula, L. J. (1976). Secure computer system: Unified exposition and multics interpretation (Tech. Rep.). DTIC Document.

Brewer, D. F., \& Nash, M. J. (1989). The chinese wall security policy. In Security and privacy, 1989. proceedings., 1989 ieee symposium on (pp. 206-214).

Hsiao, Y.-C., \& Hwang, G.-H. (2010). Implementing the chinese wall security model in workflow management systems. In Parallel and distributed processing with applications (ispa), 2010 international symposium on (pp. 574-581).

Kesarwani, A., Gupta, C., Tripathi, M. M., Gupta, V., Gupta, R., \& Chaurasiya, V. K. (2011). Implementation of chinese wall model in cloud computing for enhanced security. In Emerging trends in networks and computer communications (etncc), 2011 international conference on (pp. 411-413).

Lin, T. Y. (1989). Chinese wall security policy-an aggressive model. In Computer security applications conference, 1989., fifth annual (pp. 282-289).

Lin, T. Y. (2000). Chinese wall security model and conflict analysis. In 24th international computer software and applications conference (COMPSAC 2000), 25-28 october 2000, taipei, taiwan (pp. 122-127).

Lin, T. Y. (2002). Placing the chinese walls on the boundary of conflicts - analysis of symmetric binary relations. In 26th international computer software and applications conference (COMPSAC 2002), prolonging software life:

Development and redevelopment, 26-29 august 2002, oxford, england, proceedings (pp. 966-974).

Lin, T. Y. (2003). Chinese wall security policy models: Information flows and confining trojan horses. In Data and applications security XVII: status and prospects, IFIP TC-11 WG 11.3 seventeenth annual working conference on data and application security, august4-6, 2003, estes park, colorado, USA (pp. 275-287).

Lin, T. Y. (2007). Chinese wall security policy-revisited a short proof. In Systems, man and cybernetics, $200 \%$. isic. ieee international conference on (pp. 3027-3028).

Lin, T. Y. (2015, Oct). Chinese wall security policies information flows in business cloud. In 2015 ieee international conference on big data (big data) (p. 1603-1607). doi: 10.1109/BigData.2015.7363927

Minsky, N. H. (2004). A decentralized treatment of a highly distributed chinese-wall policy. In Policies for distributed systems and networks, 2004. policy 2004. proceedings. fifth ieee international workshop on (pp. 181-184).

Pawlak, Z. (1984). On conflicts. International Journal of Man-Machine Studies, 21(2), 127-134.

Pawlak, Z. (1997). Analysis of conflicts. In 
Joint conference of information

science, research triangle park, north

carolina (pp. 350-352).

Sandhu, R. S. (1992). Lattice-based enforcement of chinese walls. Computers $\& 3$ Security, 11(8), 753-763.

Sharifi, A., \& Tripunitara, M. V. (2013). Least-restrictive enforcement of the chinese wall security policy. In Proceedings of the 18th acm symposium on access control models and technologies (pp. 61-72).

Tsai, T.-H., Chen, Y.-C., Huang, H.-C., Huang, P.-M., \& Chou, K.-S. (2011). A practical chinese wall security model in cloud computing. In Network operations and management symposium (apnoms), 2011 13th asia-pacific (pp. 1-4).

Wu, R., Ahn, G.-J., Hu, H., \& Singhal, M. (2010). Information flow control in cloud computing. In Collaborative computing: Networking, applications and worksharing (collaboratecom), 2010 6th international conference on (pp. 1-7).

Xie, X., Ray, I., Adaikkalavan, R., \& Gamble, R. (2013). Information flow control for stream processing in clouds. In Proceedings of the 18th acm symposium on access control models and technologies (pp. 89-100). 